\title{
Updating on Roles of HIV Intrinsic Factors: A Review of Their Antiviral Mechanisms and Emerging Functions
}

\author{
Sudarat Hadpech ${ }^{a}$ Sutpirat Moonmuang ${ }^{b, c}$ Koollawat Chupradit ${ }^{b, c, d}$ \\ Umpa Yasamut ${ }^{b, c, e}$ Chatchai Tayapiwatana ${ }^{b, c}$, e
}

aDivision of Pharmacology and Biopharmacy, Faculty of Pharmaceutical Sciences, Burapha University, Chon Buri, Thailand; bivision of Clinical Immunology, Department of Medical Technology, Faculty of Associated Medical Sciences, Chiang Mai University, Chiang Mai, Thailand; 'Center of Biomolecular Therapy and Diagnostic, Faculty of Associated Medical Sciences, Chiang Mai University, Chiang Mai, Thailand; dSiriraj Center for Regenerative Medicine, Faculty of Medicine Siriraj Hospital, Mahidol University, Bangkok, Thailand; ' ${ }^{\mathrm{C}}$ enter of Innovative Immunodiagnostic Development, Faculty of Associated Medical Sciences, Chiang Mai University, Chiang Mai, Thailand

\section{Keywords}

HIV restriction factors $\cdot$ HIV intrinsic factors $\cdot$ Host-HIV interaction $\cdot$ HIV-CA interactors

\begin{abstract}
Background: Host restriction factors are cellular proteins that inhibit specific steps of the viral life cycle. Since the 1970s, several new factors have been identified, including human immunodeficiency virus-1 (HIV-1) replication restriction. Evidence accumulated in the last decade has substantially broadened our understanding of the molecular mechanisms utilized to abrogate the HIV-1 life cycle. Summary: In this review, we focus on the interaction between host restriction factors participating in the early phase of HIV-1 infection, particularly CA-targeting proteins. Host factors involved in the late phase of the replication cycle, such as viral assembly and egress factors, are also described. Additionally, current reports on well-known antiviral intrinsic factors, as well as other viral restriction factors with their emerging roles, are included. Conclusion: A comprehensive under-
\end{abstract}

karger@karger.com www.karger.com/int

Karger $\stackrel{\text { ' }}{5}$

GOPEN ACCESS
(C) 2021 The Author(s)

Published by S. Karger AG, Basel

This is an Open Access article licensed under the Creative Commons Attribution-NonCommercial-4.0 International License (CC BY-NC) (http://www.karger.com/Services/OpenAccessLicense), applicable to the online version of the article only. Usage and distribution for commercial purposes requires written permission. standing of the interactions between viruses and hosts is expected to provide insight into the design of novel HIV-1 therapeutic interventions.

(C) 2021 The Author(s)

Published by S. Karger AG, Basel

\section{Introduction}

The challenge of controlling human immunodeficiency virus-1 (HIV-1) infection involves the comprehension of viral heterogeneity, a variety of cellular host ranges, routes of transmission, immunological responses, cellular cofactors, and restriction factors [1]. A cycle of viral replication necessitates a dynamic interplay between the viral components and host cell factors. Thus, it is important to uncover how HIV-1 and host proteins interact to understand HIV-1 viral pathogenesis [2]. It has been reported that multiple cellular restriction factors constitute the first line of antiviral defense by inhibiting specific steps of HIV-1 replication including entry, uncoating, reverse transcription, and budding [3]. 
The HIV-1 capsid (CA) plays a critical role in the early stages of HIV-1 infection. Generally, CA builds up the outermost layer of the viral core structure (cone-shaped). Exposure to the cellular cytoplasmic environment after entry establishes CA as a significant target for host restriction factors that act directly to block infection, such as tripartite motif (TRIM5 $\alpha$ ) and myxovirus resistance protein $2(\mathrm{MxB} / \mathrm{Mx} 2)$. There are other acknowledged host factors that play important roles in HIV-1 infection by targeting other viral components in different steps, such as the well-known apolipoprotein B mRNA-editing enzyme catalytic subunit-like $3 \mathrm{G}$ (APOBEC3G), sterile alpha motif and histidine aspartate domain-containing protein-1 (SAMHD1), and bone marrow stromal antigen 2 (tetherin/BST-2) [4-8]. More recently, studies have revealed that serine incorporator $3 / 5$ (SERINC3/5) and many members of the TRIM family exhibit different antiviral effects against HIV-1 [9-14].

Although not all viral and host factors could be included in this review, we selectively provide an understanding of the interaction between host restriction factors involved in the early phase of HIV-1 infection started from virus entry, particularly CA-targeting factors as well as host factors involved in viral assembly and egress. Our review comprises recent updates on the well-known HIV1 intrinsic factors, and other viral restriction factors with their emerging roles are mentioned.

\section{Host Restriction Factors Involved in the Early Phase of HIV-1 Infection}

\section{SERINC3/5 Inhibits HIV-1 Entry}

During host-viral interaction, host cells have developed various factors to counteract the invasion of the viruses. In the early phase of HIV-1 infection, SERINC3 and SERINC5 are known to have an antiviral function $[15,16]$. The mechanism of action of SERINC3/5 relied on that they are incorporated into the budded virions and block the HIV-1 envelope (Env) fusion and pore formation on the membrane of target cells [17-19]. The study has suggested that these proteins act by changing the conformation or clustering of unliganded Env [19] and inhibit full fusion of HIV-1 particle without targeting just 1 particular step in the viral fusion process [20]. The energy was required for each step of the viral fusion process and increased along the fusion pathway [21, 22]. Hence, the plausible antiviral mechanism of SERINE3/5 could be that SERINE3/5 increases the energy barriers to the intermediate states. Most particles were unable to undergo the final step (pore expansion). Instead, the viral particles are restricted at the intermediate states [20]. Therefore, the delivery of the viral nucleocapsid (NC) to the cytosol is prevented $[15,16]$. However, HIV-1 encodes Nef and murine leukemia viruses encode glycosylated Gag that impedes SERINC3/5 in vitro [19]. It has been demonstrated that Nef and glycosylated Gag prevent the incorporation of SERINC3/5 into HIV-1 particles to an extent that correlates with its enhancement of infectivity [16].

\section{CA-Dependent Host Intrinsic Factors}

TRIM Proteins and Their Roles in HIV-1 Restriction

TRIM proteins belong to a group of E3 ubiquitin ligases that play crucial roles in various cellular functions, including regulation and coordination of innate immunity and antiviral responses $[9,12,23]$. In the case of HIV1 infection, TRIMs act as intrinsic restriction factors by directly interacting with viral proteins, or they can indirectly inhibit the viral replication by inducing antiviral cytokine production, thereby regulating host immune responses $[9,24]$. The feature of TRIM proteins is the highly conserved RBCC motif [24-26]. The RBCC domain includes a RING E3 ligase domain, 1 or 2 B-box (B) domains, and a coiled-coil (CC) domain [23]. The RING E3 ligase domain is a zinc-binding motif that usually mediates interactions with ubiquitin-bound E2. The RING motifs consist of key cysteine and histidine residues that coordinate with 2 zinc ions, located within 10-20 amino acids of the first methionine in the N-termini of almost all TRIM proteins, facilitating protein-protein interactions $[27,28]$. The $\mathrm{B}$ domains are also zinc-binding motifs, and the consensus sequences of $\mathrm{B} 1$ and $\mathrm{B} 2$ vary among TRIM proteins [23]. Although the function of the B domain is less well characterized, studies have suggested that the domain is essential for coordinating TRIM self-association and protein-protein interactions, as well as promoting higher order TRIM oligomerization [28]. The CC domain is a hyper-helical structure that is usually located downstream of the B domain. This CC domain mediates homomeric and heteromeric interactions among TRIM members and other proteins, particularly self-association [12].

Besides the RBCC motif, the variable C-terminal region of TRIMs, which is primarily responsible for interaction with target proteins and subcellular localization, has been characterized $[23,27,29]$ and divided into more than 10 classes $[23,28,30]$. The most prevalent TRIM Cterminal domain is PRYSPRY, the so-called 30.2. Structural analysis of PRYSPRY revealed that it is a dimer in which a donor sequence from 1 molecule binds to an ac-
68

Intervirology 2022;65:67-79

DOI: $10.1159 / 000519241$
Hadpech/Moonmuang/Chupradit/

Yasamut/Tayapiwatana 
ceptor sequence from another molecule to form a putative binding site, much like the antigen-antibody interaction [23].

TRIM5 $\alpha$ is one of the best-characterized TRIMs that present a restriction function against HIV-1 and other retroviruses [31,32]. Several mechanisms have been proposed for TRIM5 $\alpha$ antiviral function. TRIM5 $\alpha$ interacts with HIV-1 CA and plays a role in viral restriction, including dimerization, oligomerization, and ubiquitination [33-35].

Binding of TRIM5 $a$ to the CA core through higher order hexameric interactions prematurely uncoats the viral core and induces the untimely release of the viral genome $[9,36,37]$. The CA recognition site is mediated by the PRYSPRY domain [38-41], and its 4 flexible loops are believed to be important for CA pattern sensing [39, 40, $42,43]$. Although the precise molecular mechanism is not yet completely understood, a recent study indicated that TRIM5 $\alpha$ binding to CA induces global rigidification and perturbs key intermolecular interfaces necessary for higher order CA assembly, NTD-NTD and NTD-CTD intrahexameric dimer interfaces, and CTD-CTD interhexameric dimer interfaces $[44,45]$. Upon binding to the CA core, higher order oligomers of TRIM5a spontaneously assemble into a hexagonal structure on the surface of the CA [46]. TRIM5 $\alpha$ primarily forms dimeric complexes, mediated by interactions between 2 antiparallel $a$-helices that comprise the CC domain. The B domain caps the $\mathrm{N}$-terminus of each $\alpha$-helix and subsequently mediates interactions between TRIM5 $a$ dimers. This dimer interaction via $B$ domain occurs in a layered fashion with electrostatic interactions sandwiching a hydrophobic core to produce a 3-fold symmetric trimer-of-dimer structure $[46,47]$. Studies have suggested that weak interactions between TRIM5 $\alpha$ and CA at the initial step of assembly are amplified by avidity effects resulting from higher order oligomerization, which positions the SPRY domains to interact with repeating structural elements on the CA surface $[43,48]$.

Moreover, emerging evidence suggests that dynamic allostery also plays a pivotal role in CA assembly and viral infectivity [40]. In addition to HIV-1, TRIM5 $a$ displays antiviral activity against specific flaviviruses, including tick-borne encephalitis virus, Kyasanar forest disease virus, and Langat virus [37]. Additionally, potential mechanisms of antiviral function that are still under investigation include TRIM5a-mediated loss of CA, which is thought to be proteasome-dependent [49], reduced reverse transcription due to premature uncoating, and activation of innate immune responses $[37,50,51]$.

Updating on Roles of HIV Intrinsic Factors
TRIM11 has also been characterized as an HIV-1 reverse transcription restriction factor, which interacts with CA-NC protein complexes, promotes premature disassembly and release of the viral genome, and reduces transduction efficiency [52]. Although promoting the premature disassembly exerts by TRIM11 is similar to that of rhesus monkey TRIM5a (TRIM $5 \alpha_{\text {rh }}$ ), the studies have shown that these factors mediate viral restriction via different mechanisms $[52,53]$. In the previous study, it suggested that TRIM11 does not impede viral DNA nuclear import as the TRIM $5 \alpha_{\mathrm{rh}}$ does. Furthermore, examining the HIV-1 transduction and reverse transcription levels disrupted by TRIM11 and TRIM5 $\alpha_{\mathrm{rh}}$ in the presence of proteasome inhibitor (MG132) was performed to distinguish the mechanism of these 2 proteins. The result showed that the restriction of TRIM $5 \alpha_{\mathrm{rh}}$ on HIV-1 transduction and reverse transcription was sensitive to MG132; by contrast, the TRIM11 was not. This suggested a different role of these 2 TRIM proteins [52]. Moreover, TRIM11 could inhibit HIV-1 long terminal repeat (LTR) activity, which is plausibly related to its antiviral effects on NF- $\mathrm{BB}$ [53]. In agreement with another study, TRIM11 is a negative regulator of RIG-I-mediated NF- $\kappa B$ activity [54]. Additionally, microtubules have been proposed to contribute to TRIM11-mediated viral restriction [23, 52].

TRIM22 restricts normal HIV-1 transcription process by regulating the effectiveness of the transcription factor Sp1 to bind to the HIV-1 LTR promoter region. However, this mechanism is not involved in the direct interactions. Therefore, the previously observed reduction in HIV-1 LTR-mediated transcription relies on additional unspecified factors [55].

TRIM28, also known as transcriptional intermediary factor $1 \beta$ and KRAB-associated protein-1, is a nuclear protein with transcriptional regulatory activity [56]. This protein, which is also referred to as a retroviral repressor, has been recently reported to inhibit HIV-1 infectivity by specifically interfering with viral integration. By binding to the acetylated viral integrase (IN) enzyme, TRIM28 induces histone deacetylase 1 (HDAC1) complex formation, resulting in IN deacetylation and reduced integration efficacy. This finding supports the investigation of the role of TRIM28 during the viral replication cycle, showing that TRIM28 downregulation enhances viral infectivity owing to a specific elevation in viral integration. Consistent with other reports, TRIM28 overexpression reduced proviral formation [57]. Moreover, it has been reported that TRIM28 is an epigenetic adaptor that recruits multiple suppressive epigenetic modifiers to the LTR of endogenous retroviruses $[58,59]$. A recent study 
reported that TRIM28 functions as a small ubiquitin-like modifier E3 ligase to the SUMOylate P-TEFb complex to significantly restrict HIV-1 gene expression and subsequently contribute to HIV-1 latency. The manipulation of TRIM28 and its consequent SUMOylation pathway could be an avenue for developing a latency-reversing agent [60]. Therefore, the different molecular mechanisms of TRIM28 inhibitory function might parallel the dual activities, including corepressor of transcriptional activity and protein activity regulator through IN acetylation [57].

TRIM34 is another member of the TRIM family, and little is known about its biological function and subcellular localization [61]. The gene encoding human TRIM34 is located on chromosomal 11p15, clustering with a group of TRIM homologous genes containing TRIM6, TRIM5, and TRIM22 [62, 63]. Previous studies have revealed that TRIM34 plays a role in antiviral action [64]. The RBCC domain from TRIM $5 \alpha_{r h}$ can be substituted by a corresponding domain of TRIM34, and the novel recombined proteins effectively suppress HIV-1 replication [65]. Nonetheless, another study reported that TRIM34 could bind the CA of HIV-1; however, it cannot suppress infection [66]. Interestingly, a recent study demonstrated that TRIM34 could block HIV-1 infection with a particular mutation in the CA domain [67]. Single amino-acid mutations in CA, such as N74D for host cleavage and polyadenylation specific factor 6 (CPSF6) and P90A for cyclophilin A (CypA), abrogate CA binding to these host factors $[68,69]$. Normally, CPSF6 binds to CA and facilitates interaction with the factors of nuclear import pathways that enhance targeting of HIV-1 integration components to the gene-rich region $[68,70,71]$. Binding of CypA to CA protects against the action of TRIM5 $\alpha[72,73]$. These CA mutations have been demonstrated to infect cells less efficiently than the wild type in some cell types, including CD4+ T cells and monocyte-derived macrophages [67, 74, 75]. Recently, an unbiased CRISPR screening approach called HIV-CRISPR was used to identify CA-targeting restriction factors that target the CA mutants, P90A and N74D. The P90A mutant was more sensitive to TRIM5 $\alpha$ restriction. The results also revealed that the N74D mutant becomes more sensitive to the action of TRIM34. This restriction effect was found to be independent of interferon (IFN) induction and occurred during the reverse transcription step. Further investigation indicated that TRIM34 requires TRIM5 $\alpha$ to inhibit HIV-1 mutant N74D [67]. Furthermore, TRIM37 exhibits antiretroviral functions, possi- bly by interfering with several steps of the HIV-1 life cycle, including decreased viral replication upon TRIM37 transient overexpression in virus-producing cells, correlation of the reduction of viral infectivity with TRIM37 virion incorporation, increased HIV-1 replication during siRNA depletion of TRIM37 expression, and reduction in viral DNA synthesis upon TRIM37 transient overexpression $[23,76]$.

\section{MxB-CA Core Interaction Prevents HIV-1 \\ Uncoating}

Myxovirus resistance proteins belong to the family of IFN-inducible factors that show a wide range of antiviral activities [77, 78]. Similar to most mammals, there are 2 myxovirus resistance genes, MX1 and MX2, which are present in humans. These 2 genes encode the IFN-inducible dynamin-like GTPases MxA and $\mathrm{MxB}$, respectively $[79,80]$. The antiviral role of MxA has been extensively studied in various types of viruses [81-84]. By contrast, $\mathrm{MxB}$ has only recently been identified as a major inhibitor of HIV-1 replication. Fluorescence microscopy studies suggest that $\mathrm{MxB}$ proteins may bind to the CA of HIV-1 based on their observed colocalization in infected cells [85-87]. In addition, utilizing the assay based on the biochemical separation of soluble CA protein from particulate $\mathrm{CA}$ cores provides information on the role of CA during infection [88]. These findings suggest a role for $\mathrm{MxB}$ in HIV-1 infection, showing that it encounters the CA core in the cytosol and stabilizes the CA shell, leading to the prevention of HIV-1 uncoating process. Therefore, viral DNA is trapped inside the core, and transportation into the nucleus is blocked before it passed through the nuclear membrane $[88,89]$.

The exploration of the MxB-binding site on the surface of HIV-1 CA demonstrated that helices 6 and 7 of the CA are essential for the $20 \mathrm{~N}$-terminal amino acids of $\mathrm{MxB}$ to interact with and facilitate the restriction [90, 91]. $\mathrm{MxB}$, a 715-amino-acid-long protein, harbors 43 residues in the $\mathrm{N}$-terminal extension that contains a nuclear localization signal, which has been found to be critical for HIV-1 restriction [89, 92]. Another finding recapitulates the demonstration that interactions between $\mathrm{MxB}$ and $\mathrm{CA}$ are dependent on the first 83 residues of $\mathrm{MxB}$ and do not require other host factors. This is consistent with a recent report in which the $\mathrm{N}$-terminal domain of $\mathrm{MxB}$ ( 91 amino acids), including residues downstream from the nuclear localization signal, confers HIV1 restriction [93]. Taken together, these data suggest that direct engagement between $\mathrm{MxB}$ and $\mathrm{CA}$ is an important step in the restriction of HIV-1. This insightful study also
Hadpech/Moonmuang/Chupradit/

Yasamut/Tayapiwatana 
revealed that $\mathrm{MxB}$ preferentially binds to $\mathrm{CA}$ assemblies but not individual CA hexamers, implying that $\mathrm{MxB}$ is similar to TRIM5 $\alpha$ in that both recognize higher order CA assemblies [94]. Through extensive biochemical and biophysical studies and molecular dynamics simulations, recent reports have defined the HIV-1 CA site recognized by the $M x B{ }^{11} R_{R R}{ }^{13}$ motif as the interface between 3 CA hexamers. This provides the information in a residue-level mapping of the HIV-1 CA lattice-sensing restriction factor [11]. Another study has revisited the importance of the MxB GTPase (G) domain, which was previously reported to play a key role in the inhibition of different viruses, for the control of HIV-1 infection. The results indicate that the domain contains a second independent CA recognition site. Interestingly, binding of $\mathrm{CA}$ to this second binding site enhances the function of $\mathrm{MxB}$. This new report suggests that the interaction of $\mathrm{MxB}$ with HIV-1 CA is multifaceted. There are dual points of contact that contribute to the complexity of MxB inhibitory effects [10].

Previously, it was shown that MxB inhibition of HIV1 is CypA dependent. CypA is a host cell factor that assists TRIM $5 \alpha_{\text {rh }}$ in inhibiting HIV-1 in Old World primate cells [95-97]. Interestingly, MxB exerts 2 different effects on HIV-1 infection, including an inhibitory effect under the condition of a CypA interaction with viral CA and a stimulatory effect in the absence of CypA-CA interaction. CypA has been reported to stabilize the HIV-1 core and, in concert with other cellular proteins such as transportin-SR2 or Tnp3, promote the HIV-1 core disassembly and regulates HIV-1 uncoating [98]. The anti-HIV-1 activity of $\mathrm{MxB}$ is greatly enhanced following the attachment of CypA. One possible explanation for this enhancement is the efficient targeting of $\mathrm{MxB}$ to HIV-1 CA by CypA. It is possible that the multimerization of $\mathrm{MxB}$ promotes the binding of CypA to HIV-1 CA, thereby changing CypA from being a cofactor to a restriction factor [99]. Extensive studies are ongoing in the interest of $\mathrm{MxB}$. A study provides evidence that MxB sensitivity depends on HIV-1 CA conformation, rather than cofactor recruitment. The same study also demonstrated that depleting CPSF6 level in the nuclear import pathway does not affect $\mathrm{MxB}$ sensitivity, but rather the $\mathrm{CA}$ mutant P90A, which suggests that the effect of CypA binding on $\mathrm{CA}$ conformation and dynamics strongly influences $\mathrm{MxB}$ sensitivity. Thus, the relationship among the CA interactors has been proposed; CypA binding provides conformational flexibility to HIV-1 CA, facilitating simultaneous evasion of $\mathrm{MxB}$ [100].

Updating on Roles of HIV Intrinsic Factors
CPSF6 and HIV-1 CA Interaction Directs Viral Components into Actively Transcribing Genes

CPSF6 is a pre-mRNA splicing factor and a member of the serine/arginine-rich protein family [101]. In HIV-1 infection, CPSF6 binds to viral CA before the preintegration complex translocates to the host cell nucleus [70, 102]. Interaction of CPSF6 with HIV-1 CA has been implicated in diverse functions during the early phase of the viral life cycle, such as uncoating, nuclear entry, and integration targeting. This molecule has emerged as a dominant player in directing HIV-1 integration into actively transcribed genes. This is supported by studies demonstrating that the disruption of CPSF6 expression results in a redistribution of HIV-1 integration sites away from gene bodies and gene-dense chromosomal regions, thus validating the key role of CPSF6 in HIV-1 integration targeting [71]. The recognition sites on HIV-1 CA that interact with CPSF6 are part of a hydrophobic pocket formed by helices 3, 4, and 5 within the N-terminal domain [70]. CA-CPSF6 binding was confirmed using CA mutants. The introduction of N74D mutations severely reduced the amount of endogenous CPSF6 in the pellet of protein pull-down assays [103]. Moreover, the single CA mutation (N74D) has been shown to affect the sensitivity of HIV-1 to the depletion of NUP358, NUP153, and TNPO3. These HIV-1 cofactors function in nuclear import by interacting with the CA protein $[60,70,104]$.

Fasciculation and Elongation Factor Zeta 1-HIV-

1 CA Interaction Promotes Viral Trafficking and Infection

Intracellular transport is an essential process in eukaryotic cell. The transportation of cellular cargo, such as vesicles, proteins, RNA, and organelles, occurs constantly in response to various signals in a highly regulated manner [105]. Microtubule networks and other associated motors play key roles in this process. Microtubules are polarized filaments where the plus ends are directed toward the cell periphery, while the minus ends are anchored at microtubule organizing centers [103]. Motormediated transportation inside the cell is involved not only in cell polarization, migration, and transportation of cargo but also in infection by a variety of pathogens such as HIV-1. Once HIV-1 enters the host cell, the viral CA core must reach the nuclear compartment, where the genome of the virus can be delivered to the integration site. Free diffusion of the CA to the nucleus seems to be impossible because of the large size of the core structure, and the crowded environment within the cytoplasm cannot be excluded.

Intervirology 2022;65:67-79 
The question is how HIV-1 controls the direction of CA traffic toward the nucleus. It has been reported that HIV-1 hijacks cellular microtubule networks via both kinesin and dynein motors to achieve net retrograde movement [106]. This nuclear trafficking process requires kinesin- 1 adaptor protein, fasciculation and elongation factor zeta 1 (FEZ1). Live cell imaging studies have previously revealed that viral particles exhibit bidirectional trafficking. Notably, in the absence of FEZ1, there was no net movement to the nucleus [103, 107]. FEZ1 interacts with the tail of the kinesin- 1 heavy chain using regions 231-308 of its C-terminal CC domain [108]; however, it is not known whether FEZ1 directly interacts with CA or whether another mechanism is involved. A recent study has shown that FEZ1 can sense unique HIV1 CA patterns through highly negatively charged acidic stretches that interact with the positively charged center pore of the CA hexamer [106]. Therefore, FEZ1 acts as a bridge to associate virus particles with kinesin motors, thus promoting viral trafficking and infection. Even though the interaction was driven by electrostatic contacts with multiple charged stretches on FEZ1, binding was not observed with CA pentamers that contained similar charge characteristics, implying that the recognition of CA hexamers by FEZ1 is highly specific. Another study also proposed that the central pore of the CA hexamer is a conserved interaction hub for both small molecules and protein cofactors in the cell. The positively charged center of the CA hexamer, generated by the ring of the R 18 residue, serves as an ideal determinant for the highly negatively charged FEZ1 protein interaction [106, 109]. Altogether, FEZ1 specifically targets the conserved center pore of the CA hexamer, which is important for HIV-1 trafficking and infectivity [106].

\section{Host-Viral Protein Interaction Affecting Viral Assembly and Egress}

Membrane-Associated RING-CH (MARCH) 1/2/8

Reduce the Incorporation of Env

Traveling toward the plasma membrane does not warrant safety for HIV-1 Env. Membrane-associated RING$\mathrm{CH}$ (MARCH) $1 / 2 / 8$ are members of the MARCH family of RING finger E3 ubiquitin ligases and type I IFN-inducible proteins. These proteins play a role in the downregulation of the level of several membrane proteins, such as major histocompatibility complex II [110-114], CD86 [115], and transferrin receptor. Recently, it has been reported that MARCH8 targets HIV-1 Env and acts as a restriction factor. Previous studies have established that mutating the RING domain, such as CS and W114A mutations, abrogates the antiviral activity of MARCH8, which demonstrates its dependence on E3 ligase activity [116]. Additionally, MARCH1 and MARCH2 also play important roles in HIV-1 restriction in the same manner as MARCH8. MARCH1 and MARCH2 expressions in monocyte-derived macrophages increased after type I IFN treatment. MARCH1/2/8 expression in virus-producing cells reduces the efficiency of viral entry and downregulates the level of HIV-1 Env glycoproteins at the cell surface, resulting in a reduction in the incorporation of Env glycoproteins into virions $[117,118]$. Knockdown or knockout of MARCH8 in myeloid cells increases HIV1 infectivity. This finding implies that MARCH8 is a cellular restriction factor that restrains HIV-1 infection of macrophages and dendritic cells. Interestingly, Vpr, Nef, and $\mathrm{Vpu}$ do not antagonize MARCH proteins; however, the mechanism by which HIV-1 and other viruses, especially those that replicate in macrophages and dendritic cells, evade inhibition via MARCH1, MARCH2, and MARCH8 is yet to be determined [116].

\section{Tetherin/BST2 Traps Viral Particles at the Plasma Membrane}

Tetherin/BST2, also known as bone marrow stromal antigen 2, is encoded by the BST2 gene. It has also been designated as CD317 [119, 120]. Tetherin is constitutively expressed in lymphoid and myeloid cells in response to stimuli from the IFN pathway. The name tetherin comes from the function of the protein based on its ability to "tether" or trap newly budded viral particles on the surface of viral-producing cells, preventing viral release [121]. The discovery of tetherin as a host restriction factor revealed that viruses produce $\mathrm{Vpu}$ as an antagonist to tetherin $[39,122-126]$. Vpu utilizes the sequence in the $\mathrm{N}$-terminal region of the cytoplasmic domain of tetherin to remove tetherin from the plasma membrane and stimulate its degradation in the proteasome or lysosome. $\mathrm{Vpu}$ also displaces tetherin from the site of viral assembly without removing it from the cell surface, indicating that Vpu can mobilize multiple mechanisms to abrogate the restriction imposed on HIV-1 by tetherin. The in vitro induction of surface tetherin by IFN to levels comparable to those found during acute infection in vivo could overcome the action of $\mathrm{Vpu}$ and decrease the release of viral particles [119, 127-130]. Interestingly, increased expression of tetherin mRNA using CRISPR-mediated pinpoint activation of endogenous expression demonstrated that cells in which tetherin expression was highly enhanced
72

Intervirology 2022;65:67-79 DOI: $10.1159 / 000519241$
Hadpech/Moonmuang/Chupradit/

Yasamut/Tayapiwatana 
showed effective inhibition of HIV-1 production and replication even in the presence of the viral antagonist $\mathrm{Vpu}$ against tetherin. These findings confirm that the physiological stoichiometry between host restriction factors and viral antagonists may determine the outcome of the battle with viruses [131].

\section{Guanylate-Binding Proteins 2 and 5 Inhibit the}

Maturation of HIV-1 Env

Infection with HIV-1 and other viral pathogens triggers IFN production, which induces an antiviral cellular state by upregulating the expression of several of interferon-stimulated genes. The products of these genes exert numerous effector functions and may target every step of the viral replication cycle. The earliest identified interferon-stimulated genes were guanylate-binding proteins (GBPs) $[132,133]$. The human genome harbors 7 members of this protein family (GBP1-7), all of which are assumed to act as GTPases that hydrolyze GTP to GDP and GMP [134]. GBPs were initially described as cellular factors providing resistance against bacterial and protozoan pathogens [134] and were subsequently shown to be the key components of the immune defense against viruses. For instance, GBP1 has been shown to restrict vesicular stomatitis virus, encephalomyocarditis virus, and hepatitis $\mathrm{C}$ virus; however, the mechanisms of restriction have not yet been elucidated $[135,136]$. In addition, genomewide association studies have suggested a potential role for porcine GBPs in resistance to porcine reproduction and respiratory syndrome virus infection [137]. GBP2 and 5, which are highly expressed in primary monocytederived macrophages and activated CD4 T cells, have been recently shown to reduce virion infectivity by inhibiting furin function and consequently altering the maturation of viral Env glycoproteins. In HIV-infected cells, furin is an enzyme that mediates the conversion of the HIV-1 Env precursor gp 160 into mature gp 120 and gp41 [138].

Therefore, the absence of furin cleavage contributes to the incorporation of nonfunctional gp 160, which impairs HIV-1 Env maturation and infectivity [138]. Antiviral activity requires Golgi localization of GBP5, but not its GTPase activity. In agreement with the abilities of GBP2 and 5 to complement each other, the combined knockdown of the 2 proteins in macrophages showed an additive effect on infectious viral production. The inhibition of furin is a sophisticated strategy to inhibit viral production because viruses may not be able to develop resistance mutations against GBP2 and 5, as there is no direct interaction between the restriction factors and the viral com-

Updating on Roles of HIV Intrinsic Factors ponents. Therefore, GBP2 and 5 are important cell-autonomous effectors of the innate antiretroviral immune response. Nonetheless, it is tempting to speculate that viruses use several other mechanisms to overcome GBPmediated restriction. HIV-1 contains acquired mutations in its Vpu gene, thereby reducing susceptibility to GBP5 by enhancing the translation of the viral Env protein [139]. Because Vpu and Env are synthesized from a single bicistronic mRNA, shutting down Vpu expression results in increased Env expression. This mechanism confers partial resistance to GBP5 [140, 141].

\section{Endosomal Sorting Machinery and Tumor}

Susceptibility Gene 101: Roles in Viral Particle Release

Endosomal sorting complexes required for transport (ESCRT) machinery is a cytosolic protein complex consisting of ESCRT-0, ESCRT-I, ESCRT-II, and ESCRT-III [142-144]. The ESCRT machinery plays an important role in several cellular processes including multivesicular body (MVB) biogenesis, cellular abscission as well as viral budding $[144,145]$. In cytokinesis pathway, the ESCRT functions in the scission of membrane neck connecting 2 daughter cells $[144,146]$. Also, ESCRT functions in promoting the cellular vesicles budding into late endosomes to form MVB [147-149]. Additionally, in a viral infection such as HIV-1, it hijacks and uses ESCRT machinery to promote viral budding and release of newly assembled virions [150-153]. This process requires other cellular proteins such as tumor susceptibility gene 101 (Tsg101) and ALG-2 apoptosis-linked gene 2-interacting protein $\mathrm{X}$. Tsg101 functions in the cellular vacuolar protein sorting pathways, where it participates in selecting cargo for incorporation into vesicles that bud into the maturing endosomes to create intracellular compartments, which then undergo fusion with lysosomes and send their cargo for degradation. Studies have reported that the GagTsg101 interaction, especially for viral release, requires the Pro-Thr-Ala-Pro motif in Gag and a bifurcated binding groove in Tsg101. A normal level of Tsg101 is required for the budding of particles. The inhibition of Tsg101 synthesis or overexpression of the gene severely impairs HIV-1 production by arresting the release of new virions from the membrane of producer cells [151, 154, 155].

Additionally, mutations in the motifs of Tsg101 or Gag in the region responsible for interactions reduce the production of infectious particles. This indicates that the Tsg101-Gag complex is a potential target for antiviral therapy $[150,151,156]$. The ESCRT proteins, Tsg101, and apoptosis-linked gene 2 -interacting protein $\mathrm{X}$ are 


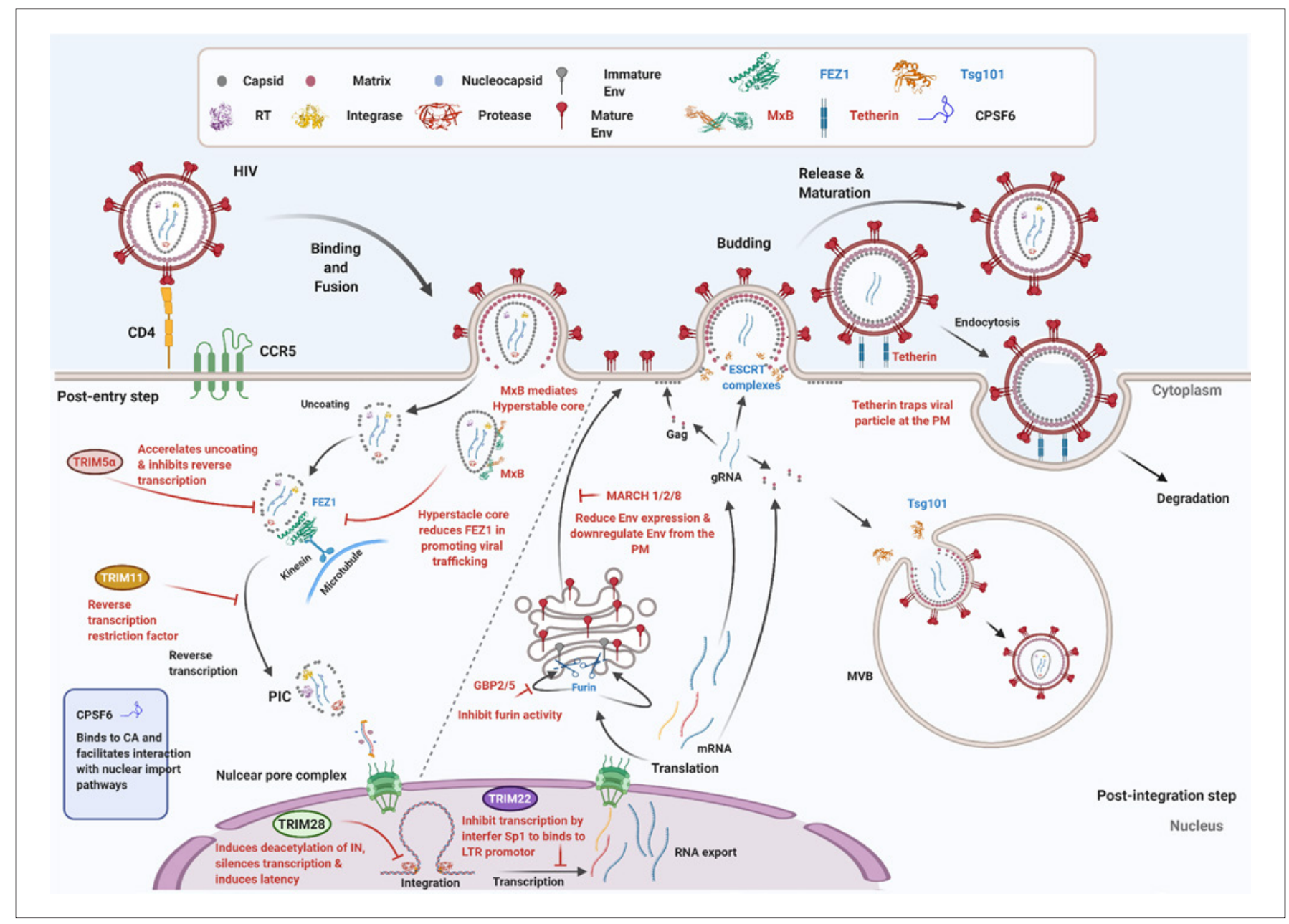

Fig. 1. The role of host factors in HIV-1 replication. HIV-1 life cycle starts from the upper left side of the image with the infection of the susceptible target cell, while the upper right side shows the ending of the replication cycle by viral egress and the budding of viral particles into a MVB. The dashed line separates the viral replication cycle into postentry and post-integration steps, the black arrows indicate the normal viral life cycle, and the red arrows indicate the inhibitory effects of viral restriction factors. The colorcoded names are based on molecule function; red indicates viral restriction factors, while blue indicates cellular factors that aid viral replication. TRIMs are the viral restriction factors depicted by the oval shapes in different colors. The sources of the protein structures used in this figure include $\mathrm{MxB}$ (PDB: 4WHJ), RT (PDB: 1REV), IN (PDB: 3LPU), and PR (PDB: 1MDP). This figure was created using BioRender.com. HIV-1, human immunodeficiency virus-1; CA, capsid; NC, nucleocapsid; Env, envelope; MxB, myxovirus resistance protein 2; Tsg101, tumor susceptibility gene 101; CPSF6, cleavage and polyadenylation specific factor 6; ESCRT, endosomal sorting complexes required for transport; TRIM $5 a$, tripartite motif; MARCH, membrane-associated RING-CH; IFN, interferon; MVB, multivesicular body; IN, integrase. known to bind to the Gag C-terminal p6 peptide. As mentioned above, Tsg101 binding is important for efficient HIV-1 release; however, how ESCRT contributes to the budding process and how their activity is coordinated with Gag assembly remain poorly understood. A recent study developed a yeast model for ESCRT-dependent Gag release. Using this system, a previously unknown interaction between the ESCRT proteins and the Gag N- terminal protein region was identified. They analyzed the Gag plasma membrane association and Gag release with Gag mutants and ESCRT knockout strains. The results suggest a transient ESCRT-matrix interaction that is replaced by Gag plasma membrane binding. These results further indicate that matrix interactions may block the ESCRT function [157]. 
Table 1. Summary of cellular factors involved in HIV-1 replication discussed in this review

\begin{tabular}{|c|c|c|c|c|}
\hline & Restriction factor & $\begin{array}{l}\text { Virus component(s) } \\
\text { targeted }\end{array}$ & Mechanism(s) of restriction & References \\
\hline 1 & TRIM5a & CA & Binds to $C A$ and induces premature uncoating & $9,36,37$ \\
\hline 2 & TRIM11 & CA-NC complex & $\begin{array}{l}\text { Interacts with CA-NC complexes, promotes premature disassembly and release of } \\
\text { viral genome, and reduces transduction efficiency }\end{array}$ & 52 \\
\hline 3 & TRIM22 & $\begin{array}{l}\text { Sp1 binding to LTR } \\
\text { promoter }\end{array}$ & $\begin{array}{l}\text { Regulates the effectiveness of the transcription factor Sp1 to bind to the HIV-1 LTR } \\
\text { promoter region }\end{array}$ & 55 \\
\hline 4 & TRIM28 & HDAC1 complex, IN & $\begin{array}{l}\text { Induces HDAC1 complex formation, resulting in IN deacetylation, and reduces } \\
\text { integration efficacy }\end{array}$ & 57 \\
\hline 5 & TRIM34 & CA & $\begin{array}{l}\text { Binds to CA and cooperates with TRIM5a to block the HIV-1 mutant (CA mutant } \\
\text { N74D) }\end{array}$ & 67 \\
\hline 6 & TRIM37 & - & Exhibits antiretroviral functions possibly by reducing viral DNA synthesis & 76 \\
\hline 10 & MARCH 1/2/8 & & $\begin{array}{l}\text { Reduces the efficiency of viral entry and downregulates the levels of HIV-1 Env } \\
\text { glycoproteins at the cell surface }\end{array}$ & $116-118$ \\
\hline 11 & Tetherin & Viral particle & $\begin{array}{l}\text { Prevents viral release by tethering budding progeny virions to the plasma membrane } \\
\text { of an infected cell }\end{array}$ & 119,127 \\
\hline 12 & GBP $2 / 5$ & Furin & $\begin{array}{l}\text { Reduces virion infectivity by inhibiting furin function and consequently reduces the } \\
\text { maturation of Env gp160 to gp120 and gp } 40\end{array}$ & 138 \\
\hline 13 & ESCRT and Tsg101 & Gag C-terminal p6 & $\begin{array}{l}\text { Promotes the budding of cellular vesicles into late endosomes to form MVBs } \\
\text { Mutations in the motifs of Tsg101 or Gag in the region responsible for interactions } \\
\text { reduce the production of infectious particles }\end{array}$ & $\begin{array}{l}150,151 \\
156\end{array}$ \\
\hline
\end{tabular}

NC, nucleocapsid; HDAC1, histone deacetylase 1; HIV-1, human immunodeficiency virus-1; TRIM5a, tripartite motif; MxB, myxovirus resistance protein 2; CPSF6, cleavage and polyadenylation specific factor 6; MARCH, membrane-associated RING-CH; GBP, guanylate-binding proteins; ESCRT, endosomal sorting complexes required for transport; Tsg101, tumor susceptibility gene 101; IN, integrase; CA, capsid; LTR, long terminal repeat; Env, envelope; MVBs, multivesicular bodies.

\section{Conclusion}

Throughout evolutionary timescales, HIV-1 and its hosts have been locked in a constant struggle for survival. This has resulted in HIV-1 evolving the capability to exploit a multitude of cellular proteins to promote its replication cycle. Reciprocally, this has led to the host cell developing restriction factors to fight back. The candidate cellular factors reviewed here are molecules that influence the postentry step of the viral life cycle, particularly CA interactors. Additionally, some other key factor actions in the post-integration steps are included. Our review incorporates recent findings on well-characterized restriction proteins and some other proteins with their emerging roles (shown in Fig. 1 and Table 1). Understanding the strategies employed by HIV-1 to gain control of the host cell and host cell mechanisms in response to its infection could open an exciting new area of research with potential translation into the design of novel antiviral drugs and treatment strategies.

\section{Conflict of Interest Statement}

The authors have no conflicts of interest to declare.

\section{Funding Sources}

This work was supported by: the National Science and Technology Development Agency, Thailand; National Research University project under Thailand's Office of the Higher Education Commission; Distinguished Research Professor Grant (NRCT 
808/2563) of the National Research Council of Thailand; National Research and Innovation Council, Thailand; Office of National Higher Education Science Research; Innovation Policy Council; The Program Management Unit for Human Resources \& Institutional Development Research and Innovation (Grant number B05F630102); and Faculty of Pharmaceutical Sciences, Burapha University.

\section{Author Contributions}

S.H. drafted the original manuscript; S.H. and S.M. created the graphic summary; S.H., C.T., U.Y., K.C., and S.M. reviewed and edited the manuscript; C.T. performed supervision. All authors have read and agreed to publish this version of the manuscript.

\section{References}

1 Levy JA. Virus-host interactions in HIV pathogenesis: directions for therapy. Adv Dent Res. 2011;23(1):13-8.

2 Tough RH, McLaren PJ. Interaction of the host and viral genome and their influence on HIV disease. Front Genet. 2018;9:720.

3 Bergantz L, Subra F, Deprez E, Delelis O, Richetta C. Interplay between intrinsic and innate immunity during HIV infection. Cells. 2019;8(8):922.

4 Olson ME, Harris RS, Harki DA. APOBEC enzymes as targets for virus and cancer therapy. Cell Chem Biol. 2018;25(1):36-49.

5 Colomer-Lluch M, Ruiz A, Moris A, Prado JG. Restriction factors: from intrinsic viral restriction to shaping cellular immunity against HIV-1. Front Immunol. 2018;9:2876.

6 Ghimire D, Rai M, Gaur R. Novel host restriction factors implicated in HIV-1 replication. J Gen Virol. 2018;99(4):435-46.

7 Malim MH, Bieniasz PD. HIV restriction factors and mechanisms of evasion. Cold Spring Harb Perspect Med. 2012;2(5):a006940.

8 DU V, De Crignis E, Re MC. Host restriction factors and human immunodeficiency virus (HIV-1): a dynamic interplay involving all phases of the viral life cycle. Curr HIV Res. 2018;16(3):184-207.

9 Giraldo MI, Hage A, van Tol S, Rajsbaum R. TRIM proteins in host defense and viral pathogenesis. Curr Clin Microbiol Rep. 2020: 1-14.

10 Betancor G, Dicks MDJ, Jimenez-Guardeño JM, Ali NH, Apolonia L, Malim MH. The GTPase domain of MX2 interacts with the HIV-1 capsid, enabling its short isoform to moderate antiviral restriction. Cell Rep. 2019;29(7): 1923-e3.

11 Smaga SS, Xu C, Summers BJ, Digianantonio KM, Perilla JR, Xiong Y. MxB restricts HIV-1 by targeting the tri-hexamer interface of the viral capsid. Structure. 2019;27(8):1234-e5.

12 Ozato K, Shin DM, Chang TH, Morse HC 3rd. TRIM family proteins and their emerging roles in innate immunity. Nat Rev Immunol. 2008;8(11):849-60.

13 Sharma S, Lewinski MK, Guatelli J. An N-glycosylated form of SERINC5 is specifically incorporated into HIV-1 virions. J Virol. 2018; 92(22): $\mathrm{e} 00753$.

14 Gonzalez-Enriquez GV, Escoto-Delgadillo M, Vazquez-Valls E, Torres-Mendoza BM. SERINC as a restriction factor to inhibit viral infectivity and the interaction with HIV. J Immunol Res. 2017;2017:1548905.
15 Rosa A, Chande A, Ziglio S, De Sanctis V, Bertorelli R, Goh SL, et al. HIV-1 Nef promotes infection by excluding SERINC5 from virion incorporation. Nature. 2015;526(7572):212-7.

16 Usami Y, Wu Y, Göttlinger HG. SERINC3 and SERINC5 restrict HIV-1 infectivity and are counteracted by Nef. Nature. 2015; 526(7572):218-23.

17 Sood C, Marin M, Chande A, Pizzato M, Melikyan GB. SERINC5 protein inhibits HIV-1 fusion pore formation by promoting functional inactivation of envelope glycoproteins. J Biol Chem. 2017;292(14):6014-26.

18 Trautz B, Wiedemann H, Lüchtenborg C, Pierini V, Kranich J, Glass B, et al. The hostcell restriction factor SERINC5 restricts HIV1 infectivity without altering the lipid composition and organization of viral particles. J Biol Chem. 2017;292(33):13702-13.

19 Timilsina U, Umthong S, Lynch B, Stablewski A, Stavrou S. SERINC5 potently restricts retrovirus infection in vivo. mBio. 2020;11(4): e00588.

20 Ward AE, Kiessling V, Pornillos O, White JM, Ganser-Pornillos BK, Tamm LK. HIV-cell membrane fusion intermediates are restricted by Serincs as revealed by cryo-electron and TIRF microscopy. J Biol Chem. 2020;295(45): 15183-95.

21 Cohen FS, Melikyan GB. The energetics of membrane fusion from binding, through hemifusion, pore formation, and pore enlargement. J Membr Biol. 2004;199(1):1-14.

22 Kawamoto S, Shinoda W. Free energy analysis along the stalk mechanism of membrane fusion. Soft Matter. 2014;10(17):3048-54.

23 van Tol S, Hage A, Giraldo MI, Bharaj P, Rajsbaum R. The trimendous Role of TRIMs in virus-host interactions. Vaccines. 2017;5(3):23.

24 van Gent M, Sparrer KMJ, Gack MU. TRIM proteins and their roles in antiviral host defenses. Annu Rev Virol. 2018;5(1):385-405.

25 Meroni G, Diez-Roux G. TRIM/RBCC, a novel class of "single protein RING finger" E3 ubiquitin ligases. Bioessays. 2005;27(11): 1147-57.

26 James LC, Keeble AH, Khan Z, Rhodes DA, Trowsdale J. Structural basis for PRYSPRYmediated tripartite motif (TRIM) protein function. Proc Natl Acad Sci USA. 2007; 104(15):6200-5.

27 Ebner P, Versteeg GA, Ikeda F. Ubiquitin enzymes in the regulation of immune responses. Crit Rev Biochem Mol Biol. 2017;52(4):42560.
28 Esposito D, Koliopoulos MG, Rittinger K. Structural determinants of TRIM protein function. Biochem Soc Trans. 2017;45(1): 183-91.

29 Short KM, Cox TC. Subclassification of the RBCC/TRIM superfamily reveals a novel motif necessary for microtubule binding. J Biol Chem. 2006;281(13):8970-80.

30 Reymond A, Meroni G, Fantozzi A, Merla G, Cairo S, Luzi L, et al. The tripartite motif family identifies cell compartments. EMBO J. 2001;20(9):2140-51.

31 Skorupka KA, Roganowicz MD, Christensen DE, Wan Y, Pornillos O, Ganser-Pornillos BK. Hierarchical assembly governs TRIM5a recognition of HIV-1 and retroviral capsids. Sci Adv. 2019;5(11):eaaw3631.

32 Nakayama EE, Shioda T. TRIM5 $\alpha$ and species tropism of HIV/SIV. Front Microbiol. 2012;3: 13.

33 Fletcher AJ, Christensen DE, Nelson C, Tan $\mathrm{CP}$, Schaller T, Lehner PJ, et al. TRIM5 $\alpha$ requires Ube2W to anchor Lys63-linked ubiquitin chains and restrict reverse transcription. EMBO J. 2015;34(15):2078-95.

34 Lamichhane R, Mukherjee S, Smolin N, Pauszek RF 3rd, Bradley M, Sastri J, et al. Dynamic conformational changes in the rhesus TRIM5 $a$ dimer dictate the potency of HIV-1 restriction. Virology. 2017;500:161-8.

35 Li YL, Chandrasekaran V, Carter SD, Woodward CL, Christensen DE, Dryden KA, et al. Primate TRIM5 proteins form hexagonal nets on HIV-1 capsids. Elife. 2016;5:e16269.

36 Ganser-Pornillos BK, Pornillos O. Restriction of HIV-1 and other retroviruses by TRIM5. Nat Rev Microbiol. 2019;17(9):546-56.

37 Chiramel AI, Meyerson NR, McNally KL, Broeckel RM, Montoya VR, Méndez-Solís O, et al. TRIM5 $a$ restricts flavivirus replication by targeting the viral protease for proteasomal degradation. Cell Rep. 2019;27(11):3269e6.

38 Roganowicz MD, Komurlu S, Mukherjee S, Plewka J, Alam SL, Skorupka KA, et al. TRIM5 $\alpha$ SPRY/coiled-coil interactions optimize avid retroviral capsid recognition. PLoS Pathog. 2017;13(10):e1006686.

39 McNatt MW, Zang T, Hatziioannou T, Bartlett M, Fofana IB, Johnson WE, et al. Species-specific activity of HIV-1 Vpu and positive selection of tetherin transmembrane domain variants. PLoS Pathog. 2009;5(2): e1000300.

Hadpech/Moonmuang/Chupradit/

Yasamut/Tayapiwatana
$76 \quad$ Intervirology 2022;65:67-79 DOI: $10.1159 / 000519241$

Yasamut/Tayapiwatana 
40 Quinn CM, Wang M, Fritz MP, Runge B, Ahn $\mathrm{J}, \mathrm{Xu} \mathrm{C}$, et al. Dynamic regulation of HIV-1 capsid interaction with the restriction factor TRIM5 $a$ identified by magic-angle spinning NMR and molecular dynamics simulations. Proc Natl Acad Sci USA. 2018;115(45): 11519-24.

41 Maillard PV, Ecco G, Ortiz M, Trono D. The specificity of TRIM5 alpha-mediated restriction is influenced by its coiled-coil domain. J Virol. 2010;84(11):5790-801

42 Biris N, Yang Y, Taylor AB, Tomashevski A, Guo M, Hart PJ, et al. Structure of the rhesus monkey TRIM5 a PRYSPRY domain, the HIV capsid recognition module. Proc Natl Acad Sci USA. 2012;109(33):13278-83.

43 Wagner JM, Christensen DE, Bhattacharya A, Dawidziak DM, Roganowicz MD, Wan Y, et al. General model for retroviral capsid pattern recognition by TRIM5 proteins. J Virol. 2018; 92(4): $\mathrm{e} 01563$.

44 Yang H, Ji X, Zhao G, Ning J, Zhao Q, Aiken C, et al. Structural insight into HIV-1 capsid recognition by rhesus TRIM5a. Proc Natl Acad Sci USA. 2012;109(45):18372-7.

45 Morger D, Zosel F, Bühlmann M, Züger S, Mittelviefhaus M, Schuler B, et al. The threefold axis of the HIV-1 capsid lattice is the species-specific binding interface for TRIM5 $\alpha$. J Virol. 2018;92(5):e01541.

46 Yu A, Skorupka KA, Pak AJ, Ganser-Pornillos BK, Pornillos O, Voth GA. TRIM5 $a$ self-assembly and compartmentalization of the HIV-1 viral capsid. Nat Commun. 2020; 11(1):1307.

47 Wagner JM, Roganowicz MD, Skorupka K, Alam SL, Christensen D, Doss G, et al. Mechanism of B-box 2 domain-mediated higherorder assembly of the retroviral restriction factor TRIM5 $\alpha$. Elife. 2016;5:e16309.

48 LiX, Sodroski J. The TRIM5alpha B-box 2 domain promotes cooperative binding to the retroviral capsid by mediating higher-order self-association. J Virol. 2008;82(23):11495502.

49 Wu X, Anderson JL, Campbell EM, Joseph AM, Hope TJ. Proteasome inhibitors uncouple rhesus TRIM5alpha restriction of HIV-1 reverse transcription and infection. Proc Natl Acad Sci USA. 2006;103(19):7465-70.

50 Mandell MA, Jain A, Arko-Mensah J, Chauhan S, Kimura T, Dinkins C, et al. TRIM proteins regulate autophagy and can target autophagic substrates by direct recognition. Dev Cell. 2014;30(4):394-409.

51 Campbell EM, Weingart J, Sette P, Opp S, Sastri J, O'Connor SK, et al. TRIM5 $\alpha$-mediated ubiquitin chain conjugation is required for inhibition of HIV-1 reverse transcription and capsid destabilization. J Virol. 2016;90(4): 1849-57.

52 Yuan T, Yao W, Tokunaga K, Yang R, Sun B. An HIV-1 capsid binding protein TRIM11 accelerates viral uncoating. Retrovirology. 2016;13(1):72.
53 Yuan T, Yao W, Huang F, Sun B, Yang R. The human antiviral factor TRIM11 is under the regulation of HIV-1 Vpr. PLoS One. 2014; 9(8):e104269.

54 Lee Y, Song B, Park C, Kwon KS. TRIM11 negatively regulates IFN $\beta$ production and antiviral activity by targeting TBK1. PLoS One. 2013;8(5):e63255.

55 Turrini F, Marelli S, Kajaste-Rudnitski A, Lusic M, Van Lint C, Das AT, et al. HIV-1 transcriptional silencing caused by TRIM22 inhibition of Sp1 binding to the viral promoter. Retrovirology. 2015;12:104.

56 Bunch H, Calderwood SK. TRIM28 as a novel transcriptional elongation factor. BMC Mol Biol. 2015;16:14.

57 Allouch A, Di Primio C, Alpi E, Lusic M, Arosio D, Giacca M, et al. The TRIM family protein KAP1 inhibits HIV-1 integration. Cell Host Microbe. 2011;9(6):484-95.

58 Boisset JC, van Cappellen W, Andrieu-Soler C, Galjart N, Dzierzak E, Robin C. In vivo imaging of haematopoietic cells emerging from the mouse aortic endothelium. Nature. 2010; 464(7285):116-20.

59 Wolf D, Goff SP. TRIM28 mediates primer binding site-targeted silencing of murine leukemia virus in embryonic cells. Cell. 2007; 131(1):46-57.

60 Ma X, Yang T, Luo Y, Wu L, Jiang Y, Song Z, et al. TRIM28 promotes HIV-1 latency by SUMOylating CDK9 and inhibiting P-TEFb. Elife. 2019;8:e42426.

61 An X, Ji B, Sun D. TRIM34 localizes to the mitochondria and mediates apoptosis through the mitochondrial pathway in HEK293T cells. Heliyon. 2020;6(1):e03115.

62 Rajsbaum R, García-Sastre A, Versteeg GA. TRIMmunity: the roles of the TRIM E3-ubiquitin ligase family in innate antiviral immunity. J Mol Biol. 2014;426(6):1265-84.

63 Sawyer SL, Emerman M, Malik HS. Discordant evolution of the adjacent antiretroviral genes TRIM22 and TRIM5 in mammals. PLoS Pathog. 2007;3(12):e197.

64 Turrini F, Di Pietro A, Vicenzi E. Lentiviral effector pathways of TRIM proteins. DNA Cell Biol. 2014;33(4):191-7.

65 Li X, Li Y, Stremlau M, Yuan W, Song B, Perron $\mathrm{M}$, et al. Functional replacement of the RING, B-box 2, and coiled-coil domains of tripartite motif 5alpha (TRIM5alpha) by heterologous TRIM domains. J Virol. 2006; 80(13):6198-206.

66 Yang Y, Brandariz-Nuñez A, Fricke T, Ivanov DN, Sarnak Z, Diaz-Griffero F. Binding of the rhesus TRIM5 a PRYSPRY domain to capsid is necessary but not sufficient for HIV-1 restriction. Virology. 2014;448:217-28.

67 Ohainle M, Kim K, Komurlu Keceli S, Felton A Campbell E, Luban J, et al. TRIM34 restricts HIV-1 and SIV capsids in a TRIM5 $\alpha$-dependent manner. PLoS Pathog. 2020;16(4):e1008507.

68 Lee K, Ambrose Z, Martin TD, Oztop I, Mulky A, Julias JG, et al. Flexible use of nuclear import pathways by HIV-1. Cell Host Microbe. 2010;7(3):221-33.
69 Schaller T, Ocwieja KE, Rasaiyaah J, Price AJ, Brady TL, Roth SL, et al. HIV-1 capsid-cyclophilin interactions determine nuclear import pathway, integration targeting and replication efficiency. PLoS Pathog. 2011;7(12): e1002439.

70 Price AJ, Fletcher AJ, Schaller T, Elliott T, Lee $\mathrm{K}$, KewalRamani VN, et al. CPSF6 defines a conserved capsid interface that modulates HIV-1 replication. PLoS Pathog. 2012;8(8): e1002896.

71 Sowd GA, Serrao E, Wang H, Wang W, Fadel HJ, Poeschla EM, et al. A critical role for alternative polyadenylation factor CPSF6 in targeting HIV-1 integration to transcriptionally active chromatin. Proc Natl Acad Sci USA. 2016;113(8):E1054-63.

72 Luban J, Bossolt KL, Franke EK, Kalpana GV, Goff SP. Human immunodeficiency virus type $1 \mathrm{Gag}$ protein binds to cyclophilins A and B. Cell. 1993;73(6):1067-78.

73 Kim K, Dauphin A, Komurlu S, McCauley SM, Yurkovetskiy L, Carbone C, et al. Cyclophilin A protects HIV-1 from restriction by human TRIM5 $\alpha$. Nat Microbiol. 2019; 4(12):2044-51.

74 Ambrose Z, Lee K, Ndjomou J, Xu H, Oztop I, Matous J, et al. Human immunodeficiency virus type 1 capsid mutation N74D alters cyclophilin A dependence and impairs macrophage infection. J Virol. 2012;86(8):4708-14.

75 Bulli L, Apolonia L, Kutzner J, Pollpeter D, Goujon C, Herold N, et al. Complex interplay between HIV-1 capsid and MX2-independent alpha interferon-induced antiviral factors. J Virol. 2016;90(16):7469-80.

76 Tabah AA, Tardif K, Mansky LM. AntiHIV-1 activity of Trim 37. J Gen Virol. 2014; 95(Pt 4):960-7.

77 Fricke T, White TE, Schulte B, de Souza Aranha Vieira DA, Dharan A, Campbell EM, et al. $\mathrm{MxB}$ binds to the HIV-1 core and prevents the uncoating process of HIV-1. Retrovirology. 2014;11:68

78 Mitchell PS, Emerman M, Malik HS. An evolutionary perspective on the broad antiviral specificity of MxA. Curr Opin Microbiol. 2013;16(4):493-9.

79 Horisberger MA, Staeheli P, Haller O. Interferon induces a unique protein in mouse cells bearing a gene for resistance to influenza virus. Proc Natl Acad Sci USA. 1983;80(7):1910-4.

80 Aebi M, Fäh J, Hurt N, Samuel CE, Thomis D, Bazzigher L, et al. cDNA structures and regulation of two interferon-induced human $\mathrm{Mx}$ proteins. Mol Cell Biol. 1989;9(11):5062-72.

81 Xiao H, Killip MJ, Staeheli P, Randall RE, Jackson D. The human interferon-induced MxA protein inhibits early stages of influenza A virus infection by retaining the incoming viral genome in the cytoplasm. J Virol. 2013; 87(23):13053-8.

82 Matzinger SR, Carroll TD, Dutra JC, Ma ZM, Miller CJ. Myxovirus resistance gene A (MxA) expression suppresses influenza A virus replication in alpha interferon-treated primate cells. J Virol. 2013;87(2):1150-8. 
83 Netherton CL, Simpson J, Haller O, Wileman TE, Takamatsu HH, Monaghan P, et al. Inhibition of a large double-stranded DNA virus by MxA protein. J Virol. 2009;83(5):2310-20.

$84 \mathrm{Li} \mathrm{N}$, Zhang L, Chen L, Feng W, Xu Y, Chen $\mathrm{F}$, et al. MxA inhibits hepatitis B virus replication by interaction with hepatitis $B$ core antigen. Hepatology. 2012;56(3):803-11.

85 Kochs G, Janzen C, Hohenberg H, Haller O. Antivirally active MxA protein sequesters $\mathrm{La}$ Crosse virus nucleocapsid protein into perinuclear complexes. Proc Natl Acad Sci USA. 2002;99(5):3153-8.

86 Reichelt M, Stertz S, Krijnse-Locker J, Haller O, Kochs G. Missorting of LaCrosse virus nucleocapsid protein by the interferon-induced MxA GTPase involves smooth ER membranes. Traffic. 2004;5(10):772-84.

87 Liu Z, Pan Q, Ding S, Qian J, Xu F, Zhou J, et al. The interferon-inducible MxB protein inhibits HIV-1 infection. Cell Host Microbe. 2013;14(4):398-410.

88 Goujon C, Moncorgé O, Bauby H, Doyle T, Ward CC, Schaller T, et al. Human MX2 is an interferon-induced post-entry inhibitor of HIV-1 infection. Nature. 2013;502(7472): 559-62.

89 Kane M, Yadav SS, Bitzegeio J, Kutluay SB, Zang T, Wilson SJ, et al. MX2 is an interferoninduced inhibitor of HIV-1 infection. Nature. 2013;502(7472):563-6.

90 Goudreau N, Lemke CT, Faucher AM, Grand-Maitre C, Goulet S, Lacoste JE, et al. Novel inhibitor binding site discovery on HIV-1 capsid N-terminal domain by NMR and X-ray crystallography. ACS Chem Biol. 2013;8(5):1074-82.

91 Lemke CT, Titolo S, Goudreau N, Faucher AM, Mason SW, Bonneau P. A novel inhibitor-binding site on the HIV-1 capsid N-terminal domain leads to improved crystallization via compound-mediated dimerization. Acta Crystallogr D Biol Crystallogr. 2013; 69(Pt 6):1115-23.

92 Melen K, Keskinen P, Ronni T, Sareneva T, Lounatmaa K, Julkunen I. Human MxB protein, an interferon-alpha-inducible GTPase, contains a nuclear targeting signal and is localized in the heterochromatin region beneath the nuclear envelope. J Biol Chem. 1996;271(38):23478-86.

93 Goujon C, Moncorgé O, Bauby H, Doyle T, Barclay WS, Malim MH. Transfer of the amino-terminal nuclear envelope targeting domain of human MX2 converts MX1 into an HIV-1 resistance factor. J Virol. 2014;88(16): 9017-26.

94 Pertel T, Hausmann S, Morger D, Züger S, Guerra J, Lascano J, et al. TRIM5 is an innate immune sensor for the retrovirus capsid lattice. Nature. 2011;472(7343):361-5.

95 Berthoux L, Sebastian S, Sokolskaja E, Luban J. Cyclophilin A is required for TRIM5 (alpha)-mediated resistance to HIV-1 in old world monkey cells. Proc Natl Acad Sci USA. 2005;102(41):14849-53.
96 Keckesova Z, Ylinen LM, Towers GJ. Cyclophilin A renders human immunodeficiency virus type 1 sensitive to Old World monkey but not human TRIM5 alpha antiviral activity. J Virol. 2006;80(10):4683-90.

97 Lin TY, Emerman M. Determinants of cyclophilin A-dependent TRIM5 alpha restriction against HIV-1. Virology. 2008; 379(2):335-41.

98 Shah VB, Shi J, Hout DR, Oztop I, Krishnan $\mathrm{L}, \mathrm{Ahn} \mathrm{J}$, et al. The host proteins transportin SR2/TNPO3 and cyclophilin A exert opposing effects on HIV-1 uncoating. J Virol. 2013;87(1):422-32.

99 Javanbakht H, Diaz-Griffero F, Yuan W, Yeung DF, Li X, Song B, et al. The ability of multimerized cyclophilin A to restrict retrovirus infection. Virology. 2007;367(1):1929.

100 Miles RJ, Kerridge C, Hilditch L, Monit C, Jacques DA, Towers GJ. MxB sensitivity of HIV-1 is determined by a highly variable and dynamic capsid surface. Elife. 2020;9: e56910.

101 Ruegsegger U, Blank D, Keller W. Human pre-mRNA cleavage factor Im is related to spliceosomal SR proteins and can be reconstituted in vitro from recombinant subunits. Mol Cell. 1998;1(2):243-53.

102 Rasheedi S, Shun MC, Serrao E, Sowd GA, Qian J, Hao C, et al. The cleavage and polyadenylation specificity factor 6 (CPSF6) subunit of the capsid-recruited pre-messenger RNA cleavage factor I (CFIm) complex mediates HIV-1 integration into genes. J Biol Chem. 2016;291(22):11809-19.

103 Malikov V, da Silva ES, Jovasevic V, Bennett G, de Souza Aranha Vieira DA, Schulte B, et al. HIV-1 capsids bind and exploit the kinesin-1 adaptor FEZ1 for inward movement to the nucleus. Nat Commun. 2015;6:6660.

104 Yamashita M, Engelman AN. Capsid-dependent host factors in HIV-1 infection. Trends Microbiol. 2017;25(9):741-55.

105 Dharan A, Campbell EM. Role of microtubules and microtubule-associated proteins in HIV-1 infection. J Virol. 2018;92(16): e00085.

106 Huang PT, Summers BJ, Xu C, Perilla JR, Malikov V, Naghavi MH, et al. FEZ1 is recruited to a conserved cofactor site on capsid to promote HIV-1 trafficking. Cell Rep. 2019;28(9):2373-e7.

107 McDonald D, Vodicka MA, Lucero G, Svitkina TM, Borisy GG, Emerman M, et al. Visualization of the intracellular behavior of HIV in living cells. J Cell Biol. 2002;159(3): 441-52.

108 Blasius TL, Cai D, Jih GT, Toret CP, Verhey KJ. Two binding partners cooperate to activate the molecular motor Kinesin-1. J Cell Biol. 2007;176(1):11-7.

109 Forshey BM, von Schwedler U, Sundquist WI, Aiken C. Formation of a human immunodeficiency virus type 1 core of optimal stability is crucial for viral replication. J Virol. 2002;76(11):5667-77.
110 Ishido S, Kajikawa M. MHC class II fine tuning by ubiquitination: lesson from MARCHs. Immunogenetics. 2019;71(3): 197-201.

111 von Rohrscheidt J, Petrozziello E, Nedjic J, Federle C, Krzyzak L, Ploegh HL, et al. Thymic CD4 T cell selection requires attenuation of March8-mediated MHCII turnover in cortical epithelial cells through CD83. J Exp Med. 2016;213(9):1685-94.

112 De Gassart A, Camosseto V, Thibodeau J, Ceppi M, Catalan N, Pierre P, et al. MHC class II stabilization at the surface of human dendritic cells is the result of maturationdependent MARCH I down-regulation. Proc Natl Acad Sci USA. 2008;105(9):34916.

113 Matsuki Y, Ohmura-Hoshino M, Goto E Aoki M, Mito-Yoshida M, Uematsu M, et al. Novel regulation of MHC class II function in B cells. EMBO J. 2007;26(3):846-54.

114 Ohmura-Hoshino M, Matsuki Y, Mito-Yoshida M, Goto E, Aoki-Kawasumi M, Nakayama $\mathrm{M}$, et al. Cutting edge: requirement of MARCH-I-mediated MHC II ubiquitination for the maintenance of conventional dendritic cells. J Immunol. 2009;183(11): 6893-7.

115 Corcoran K, Jabbour M, Bhagwandin C, Deymier MJ, Theisen DL, Lybarger L. Ubiquitin-mediated regulation of CD86 protein expression by the ubiquitin ligase membrane-associated RING-CH-1 (MARCH1). J Biol Chem. 2011;286(43):37168-80.

116 Beitari S, Wang Y, Liu SL, Liang C. HIV-1 envelope glycoprotein at the interface of host restriction and virus evasion. Viruses. 2019;11(4):311.

117 Tada T, Zhang Y, Koyama T, Tobiume M Tsunetsugu-Yokota Y, Yamaoka S, et al. MARCH8 inhibits HIV-1 infection by reducing virion incorporation of envelope glycoproteins. Nat Med. 2015;21(12):15027.

118 Zhang Y, Tada T, Ozono S, Yao W, Tanaka $\mathrm{M}$, Yamaoka S, et al. Membrane-associated RING-CH (MARCH) 1 and 2 are MARCH family members that inhibit HIV-1 infection. J Biol Chem. 2019;294(10):3397-405.

119 Evans DT, Serra-Moreno R, Singh RK, Guatelli JC. BST-2/tetherin: a new component of the innate immune response to enveloped viruses. Trends Microbiol. 2010; 18(9):388-96

120 Kuhl BD, Cheng V, Wainberg MA, Liang C. Tetherin and its viral antagonists. J Neuroimmune Pharmacol. 2011;6(2):188-201.

121 Venkatesh S, Bieniasz PD. Mechanism of HIV-1 virion entrapment by tetherin. PLoS Pathog. 2013;9(7):e1003483.

122 Dube M, Roy BB, Guiot-Guillain P, Binette J, Mercier J, Chiasson A, et al. Antagonism of tetherin restriction of HIV-1 release by $\mathrm{Vpu}$ involves binding and sequestration of the restriction factor in a perinuclear compartment. PLoS Pathog. 2010;6(4): e1000856 
123 Iwabu Y, Fujita H, Kinomoto M, Kaneko K, Ishizaka Y, Tanaka Y, et al. HIV-1 accessory protein Vpu internalizes cell-surface BST-2/ tetherin through transmembrane interactions leading to lysosomes. J Biol Chem. 2009;284(50):35060-72.

124 Vigan R, Neil SJ. Determinants of tetherin antagonism in the transmembrane domain of the human immunodeficiency virus type 1 Vpu protein. J Virol. 2010;84(24):1295870.

125 Kobayashi T, Ode H, Yoshida T, Sato K, Gee $\mathrm{P}$, Yamamoto SP, et al. Identification of amino acids in the human tetherin transmembrane domain responsible for HIV-1 Vpu interaction and susceptibility. J Virol. 2011;85(2):932-45.

126 Stremlau M, Perron M, Welikala S, Sodroski J. Species-specific variation in the B30.2 (SPRY) domain of TRIM5alpha determines the potency of human immunodeficiency virus restriction. J Virol. 2005;79(5):313945.

127 Perez-Caballero D, Zang T, Ebrahimi A, McNatt MW, Gregory DA, Johnson MC, et al. Tetherin inhibits HIV-1 release by directly tethering virions to cells. Cell. 2009; 139(3):499-511.

128 McNatt MW, Zang T, Bieniasz PD. Vpu binds directly to tetherin and displaces it from nascent virions. PLoS Pathog. 2013; 9(4):e1003299.

129 Van Damme N, Goff D, Katsura C, Jorgenson RL, Mitchell R, Johnson MC, et al. The interferon-induced protein BST-2 restricts HIV-1 release and is downregulated from the cell surface by the viral Vpu protein. Cell Host Microbe. 2008;3(4):245-52.

130 Neil SJ, Zang T, Bieniasz PD. Tetherin inhibits retrovirus release and is antagonized by HIV-1 Vpu. Nature. 2008;451(7177): 425-30.

131 Zhang Y, Ozono S, Yao W, Tobiume M, Yamaoka S, Kishigami S, et al. CRISPR-mediated activation of endogenous BST-2/tetherin expression inhibits wild-type HIV-1 production. Sci Rep. 2019;9(1):3134.

132 Cheng YS, Colonno RJ, Yin FH. Interferon induction of fibroblast proteins with guanylate binding activity. J Biol Chem. 1983; 258(12):7746-50.

133 Staeheli P, Prochazka M, Steigmeier PA, Haller O. Genetic control of interferon action: mouse strain distribution and inheritance of an induced protein with guanylatebinding property. Virology. 1984;137(1): $135-42$.
134 Vestal DJ, Jeyaratnam JA. The guanylatebinding proteins: emerging insights into the biochemical properties and functions of this family of large interferon-induced guanosine triphosphatase. J Interferon Cytokine Res. 2011;31(1):89-97.

135 Anderson SL, Carton JM, Lou J, Xing L, Rubin BY. Interferon-induced guanylate binding protein-1 (GBP-1) mediates an antiviral effect against vesicular stomatitis virus and encephalomyocarditis virus. Virology. 1999;256(1):8-14.

136 Itsui Y, Sakamoto N, Kurosaki M, Kanazawa N, Tanabe Y, Koyama T, et al. Expressional screening of interferon-stimulated genes for antiviral activity against hepatitis C virus replication. J Viral Hepat. 2006; 13(10):690-700.

137 Rowland RR, Lunney J, Dekkers J. Control of porcine reproductive and respiratory syndrome (PRRS) through genetic improvements in disease resistance and tolerance. Front Genet. 2012;3:260.

138 Braun E, Hotter D, Koepke L, Zech F, Gross $\mathrm{R}$, Sparrer KMJ, et al. Guanylate-binding proteins 2 and 5 exert broad antiviral activity by inhibiting furin-mediated processing of viral envelope proteins. Cell Rep. 2019; 27(7):2092-e10.

139 Krapp C, Hotter D, Gawanbacht A, McLaren PJ, Kluge SF, Stürzel CM, et al. Guanylate binding protein (GBP) 5 is an interferoninducible inhibitor of HIV-1 infectivity. Cell Host Microbe. 2016;19(4):504-14.

140 Guerrero S, Batisse J, Libre C, Bernacchi S, Marquet R, Paillart JC. HIV-1 replication and the cellular eukaryotic translation apparatus. Viruses. 2015;7(1):199-218.

141 Hotter D, Sauter D, Kirchhoff F. Guanylate binding protein 5 : impairing virion infectivity by targeting retroviral envelope glycoproteins. Small GTPases. 2017;8(1):31-7.

142 Schmidt O, Teis D. The ESCRT machinery. Curr Biol. 2012;22(4):R116-20.

143 Babst M. MVB vesicle formation: ESCRTdependent, ESCRT-independent and everything in between. Curr Opin Cell Biol. 2011; 23(4):452-7.

144 Wollert T, Yang D, Ren X, Lee HH, Im YJ, Hurley JH. The ESCRT machinery at a glance. J Cell Sci. 2009;122(Pt 13):2163-6.
145 Piper RC, Katzmann DJ. Biogenesis and function of multivesicular bodies. Annu Rey Cell Dev Biol. 2007;23:519-47.

146 Carlton JG, Martin-Serrano J. Parallels between cytokinesis and retroviral budding: a role for the ESCRT machinery. Science. 2007;316(5833):1908-12.

147 Henne WM, Buchkovich NJ, Emr SD. The ESCRT pathway. Dev Cell. 2011;21(1):7791.

148 Wollert T, Hurley JH. Molecular mechanism of multivesicular body biogenesis by ESCRT complexes. Nature. 2010;464(7290): 864-9.

149 Morita E. Differential requirements of mammalian ESCRTs in multivesicular body formation, virus budding and cell division. FEBS J. 2012;279(8):1399-406.

150 Martin-Serrano J, Zang T, Bieniasz PD HIV-1 and Ebola virus encode small peptide motifs that recruit Tsg 101 to sites of particle assembly to facilitate egress. Nat Med. 2001; 7(12):1313-9.

151 Garrus JE, von Schwedler UK, Pornillos OW, Morham SG, Zavitz KH, Wang HE, et al. Tsg101 and the vacuolar protein sorting pathway are essential for HIV-1 budding. Cell. 2001;107(1):55-65.

152 Trkola A. HIV-host interactions: vital to the virus and key to its inhibition. Curr Opin Microbiol. 2004;7(5):407-11.

153 Ahmed I, Akram Z, Iqbal HMN, Munn AL. The regulation of endosomal sorting complex required for transport and accessory proteins in multivesicular body sorting and enveloped viral budding: an overview. Int J Biol Macromol. 2019;127:1-11.

154 Pornillos O, Higginson DS, Stray KM, Fisher RD, Garrus JE, Payne M, et al. HIV Gag mimics the Tsg101-recruiting activity of the human Hrs protein. J Cell Biol. 2003;162(3): 425-34.

155 Goff A, Ehrlich LS, Cohen SN, Carter CA Tsg101 control of human immunodeficiency virus type $1 \mathrm{Gag}$ trafficking and release. J Virol. 2003;77(17):9173-82.

156 Liu F, Stephen AG, Fisher RJ, Burke TR Jr. Protected aminooxyprolines for expedited library synthesis: application to Tsg101-directed proline-oxime containing peptides. Bioorg Med Chem Lett. 2008;18(3):1096101.

157 Meusser B, Purfuerst B, Luft FC. HIV-1 Gag release from yeast reveals ESCRT interaction with the Gag N-terminal protein region. J Biol Chem. 2020;295(52):17950-72. 